\title{
The degree-diameter problem for sparse graph classes
}

\author{
Guillermo Pineda-Villavicencio* \\ Centre for Informatics and Applied Optimisation \\ Federation University Australia \\ Ballarat, Australia \\ work@guillermo.com.au
}

\author{
David R. Wood ${ }^{\dagger}$ \\ School of Mathematical Sciences \\ Monash University \\ Melbourne, Australia \\ david.wood@monash.edu
}

Submitted: Apr 24, 2014; Accepted: May 27, 2015; Published: Jun 15, 2015

Mathematics Subject Classifications: 05C75, 05C10, 05C83

\begin{abstract}
The degree-diameter problem asks for the maximum number of vertices in a graph with maximum degree $\Delta$ and diameter $k$. For fixed $k$, the answer is $\Theta\left(\Delta^{k}\right)$. We consider the degree-diameter problem for particular classes of sparse graphs, and establish the following results. For graphs of bounded average degree the answer is $\Theta\left(\Delta^{k-1}\right)$, and for graphs of bounded arboricity the answer is $\Theta\left(\Delta^{\lfloor k / 2\rfloor}\right)$, in both cases for fixed $k$. For graphs of given treewidth, we determine the the maximum number of vertices up to a constant factor. Other precise bounds are given for graphs embeddable on a given surface and apex-minor-free graphs.
\end{abstract}

Keywords: degree-diameter problem; treewidth; arboricity; sparse graph; surface graph; apex-minor-free graph

\section{Introduction}

Let $N(\Delta, k)$ be the maximum number of vertices in a graph with maximum degree at most $\Delta$ and diameter at most $k$. Determining $N(\Delta, k)$ is called the degree-diameter problem and is widely studied, especially motivated by questions in network design; see [22] for a survey. Obviously, $N(\Delta, k)$ is at most the number of vertices at distance at most $k$ from a fixed vertex. For $\Delta \geqslant 3$ (which we implicitly assume), it follows that

$$
N(\Delta, k) \leqslant M(\Delta, k):=1+\Delta \sum_{i=0}^{k-1}(\Delta-1)^{i}=\frac{\Delta(\Delta-1)^{k}-2}{\Delta-2} .
$$

${ }^{*}$ Research supported by a postdoctoral fellowship funded by the Skirball Foundation via the Center for Advanced Studies in Mathematics at Ben-Gurion University of the Negev, and by an ISF grant. Corresponding author.

${ }^{\dagger}$ Research supported by the Australian Research Council. 
This inequality is called the Moore bound $[22$, p. 8]. The best lower bound is

$$
N(\Delta, k) \geqslant f(k) \Delta^{k}
$$

for some function $f$. For example, the de Bruijn graph shows that $N(\Delta, k) \geqslant\left(\frac{\Delta}{2}\right)^{k}$; see Lemma 1. Canale and Gómez [3] established the best known asymptotic bound of $N(\Delta, k) \geqslant\left(\frac{\Delta}{1.59}\right)^{k}$ for sufficiently large $\Delta$.

For a class of graphs $\mathcal{G}$, let $N(\Delta, k, \mathcal{G})$ be the maximum number of vertices in a graph in $\mathcal{G}$ with maximum degree at most $\Delta$ and diameter at most $k$. We consider $N(\Delta, k, \mathcal{G})$ for some particular classes $\mathcal{G}$ of sparse graphs, focusing on the case of small diameter $k$, and large maximum degree $\Delta$. We prove lower and upper bounds on $N(\Delta, k, \mathcal{G})$ of the form

$$
f(k) \Delta^{g(k)}
$$

for some functions $f$ and $g$. Since $k$ is assumed to be small compared to $\Delta$, the most important term in such a bound is $g(k)$. Thus our focus is on $g(k)$ with $f(k)$ a secondary concern.

We first state two straightforward examples, namely bipartite graphs and trees. The maximum number of vertices in a bipartite graph with maximum degree $\Delta$ and diameter $k$ is $f(k) \Delta^{k-1}$ for some function $f$; see references [22, Section 2.4.4] and [1, 5]. And for trees, it is easily seen that the maximum number of vertices is within a constant factor of $(\Delta-1)^{\lfloor k / 2\rfloor}$, which is a big improvement over the unrestricted bound of $\Delta^{k}$. Some of the results in this paper can be thought of as generalisations of this observation. bold.

The following table summarises our current knowledge, where original results are in

\begin{tabular}{llr}
\hline graph class & diameter $k$ & max. number of vertices \\
\hline general & & $f(k) \Delta^{k}$ \\
3-colourable & $k \geqslant 2$ & $f(k) \Delta^{k}$ \\
triangle-free 3-colourable & $k \geqslant 4$ & $f(k) \Delta^{k}$ \\
bipartite & & $f(k) \Delta^{k-1}$ \\
average degree $\boldsymbol{d}$ & & $f(k) d \Delta^{k-1}$ \\
arboricity $\boldsymbol{b}$ & odd $k$ & $f(k, b) \Delta^{\lfloor k / 2\rfloor}$ \\
treewidth $\boldsymbol{t}$ & even $k$ & $c t(\Delta-1)^{(k-1) / 2}$ \\
treewidth $\boldsymbol{t}$ & odd $k$ & $\leqslant c(g+1) k(\Delta-1)^{(k-1) / 2}$ \\
Euler genus $\boldsymbol{g}$ & even $k$ & $\leqslant c \sqrt{(g+1) k}(\Delta-1)^{k / 2}$ \\
Euler genus $\boldsymbol{g}$ & & $c \Delta \Delta^{\lfloor k / 2\rfloor}$ \\
trees &
\end{tabular}

First consider the class of graphs with average degree $d$. In this case, we prove that the maximum number of vertices is $f(k) d \Delta^{k-1}$ for some function $f$ (see Section 3). This shows that by assuming bounded average degree we obtain a modest improvement over the standard bound of $(\Delta-1)^{k}$. A much more substantial improvement is obtained by considering arboricity. 
The arboricity of a graph $G$ is the minimum number of spanning forests whose union is $G$. Nash-Williams [24] proved that the arboricity of $G$ equals

$$
\max _{H \subseteq G}\left\lceil\frac{|E(H)|}{|V(H)|-1}\right\rceil,
$$

where the maximum is taken over all subgraphs $H$ of $G$. For example, it follows from Euler's formula that every planar graph has arboricity at most 3, and every graph with Euler genus $g$ has arboricity at most $O(\sqrt{g})$. More generally, every graph that excludes a fixed minor has bounded arboricity. Note that $\delta \leqslant d \leqslant 2 b$ for every graph with minimum degree $\delta$, average degree $d$, and arboricity $b$. Arboricity is a more refined measure than average degree, in the sense that a graph has bounded arboricity if and only if every subgraph has bounded average degree.

We prove that for a graph with arboricity $b$ the maximum number of vertices is $f(b, k) \Delta^{\lfloor k / 2\rfloor}$ for some function $f$ (see Section 4 ). Thus by moving from bounded average degree to bounded arboricity the $g(k)$ term discussed above is reduced from $k-1$ to $\left\lfloor\frac{k}{2}\right\rfloor$. This result generalises the above-mentioned bound for trees, which have arboricity 1. The dependence on $b$ in $f$ can be reduced by making more restrictive assumptions about the graph.

Treewidth is a parameter that measures how tree-like a given graph is. The treewidth of a graph $G$ can be defined to be the minimum integer $t$ such that $G$ is a spanning subgraph of a chordal ${ }^{1}$ graph with no $(t+2)$-clique. For example, trees are exactly the connected graphs with treewidth 1 . See $[2,26]$ for background on treewidth. Since the arboricity of a graph is at most its treewidth, bounded treewidth is indeed a more restrictive assumption than bounded arboricity. We prove that the maximum number of vertices in a graph with treewidth $t$ is within a constant factor of $t(\Delta-1)^{(k-1) / 2}$ if $k$ is odd, and of $\sqrt{t}(\Delta-1)^{k / 2}$ if $k$ is even (and $\Delta$ is large). These results immediately imply the best known bounds for graphs of given Euler genus ${ }^{2}$, and new bounds for apex-minor-free graphs. All these results are presented in Section 5.

Our results in Section 6 are of a different nature. There, we describe (non-sparse) graph classes for which the maximum number of vertices is not much different from the unrestricted case. In particular, we prove that for $k \geqslant 2$, there are 3-colourable graphs with $f(k) \Delta^{k}$ vertices, and for for $k \geqslant 4$, there are triangle-free 3-colourable graphs with $f(k) \Delta^{k}$ vertices. These results are in contrast to the bipartite case, in which $f(k) \Delta^{k-1}$ is the answer.

All undefined terminology and notation is in reference [9].

\footnotetext{
${ }^{1} \mathrm{~A}$ graph is chordal if every induced cycle is a triangle.

${ }^{2}$ A surface is a non-null compact connected 2-manifold without boundary. Every surface is homeomorphic to the sphere with $h$ handles or the sphere with $c$ cross-caps. The sphere with $h$ handles has Euler genus $2 h$, and the sphere with $c$ cross-caps has Euler genus $c$. The Euler genus of a graph $G$ is the minimum Euler genus of a surface in which $G$ embeds. See the monograph by Mohar and Thomassen [23] for background on graphs embedded in surfaces.
} 


\section{Basic Constructions}

This section gives some graph constructions that will later be used for proving lower bounds on $N(\Delta, k, \mathcal{G})$. A digraph is a directed graph possibly with loops and possibly with arcs in opposite directions between two vertices. A digraph is $r$-inout-regular if each vertex has indegree $r$ and outdegree $r$ (where a loop at $v$ counts in the indegree and the outdegree of $v$ ). A digraph has strong diameter $k$ if for all (not necessarily distinct) vertices $v$ and $w$ there is a directed walk from $v$ to $w$ of length exactly $k$.

de Bruijn [4] and Good [16] independently introduced what is now known as the de Bruijn digraph $\vec{B}(r, k)$. Fiol et al. [14], and Zhang and Lin [32] showed that $\vec{B}(r, k)$ can be constructed recursively as a line digraph, as we now explain. If $G$ is a digraph with arc set $A(G)$, then the line digraph $L(G)$ has vertex set $A(G)$, where $(u v, v w)$ is an arc of $L(G)$ for all distinct arcs $u v, v w \in A(G)$. Let $\vec{B}(r, 1)$ be the $r$-vertex digraph in which every arc is present (including loops). Now recursively define $\vec{B}(r, k):=L(\vec{B}(r, k-1))$. Then $\vec{B}(r, k)$ has $r^{k}$ vertices, is $r$-inout-regular, and has strong diameter $k$; see [14, Sec. IV]. Define the de Bruijn graph $B(r, k)$ to be the undirected graph that underlies $\vec{B}(r, k)$ (ignoring loops, and replacing bidirectional arcs by a single edge).

Lemma 1. For all integers $r \geqslant 1$ and $k \geqslant 1$ the de Bruijn graph $B(r, k)$ has $r^{k}$ vertices, maximum degree at most $2 r$, and diameter $k$. Moreover, for $k \geqslant 2$, there are sets $B_{1}, \ldots, B_{r^{k-1}}$ of vertices in $B(r, k)$, each containing $2 r-2$ or $2 r$ vertices, such that each vertex of $B(r, k)$ is in exactly two of the $B_{i}$, and the endpoints of each edge of $B(r, k)$ are in some $B_{i}$.

Proof. Clearly $B(r, k)$ has $r^{k}$ vertices, has maximum degree at most $2 r$, and has (undirected) diameter $k$ (since loops can be ignored in shortest paths). It remains to prove the final claim of the lemma, where $k \geqslant 2$. For each vertex $v$ of $\vec{B}(r, k-1)$, let $B_{v}$ be the set of vertices of $B(r, k)$ that correspond to non-loop arcs incident with $v$ in $\vec{B}(r, k-1)$. Thus $\left|B_{v}\right|$ equals $2 r-2$ or $2 r$ depending on whether there is a loop at $v$ in $\vec{B}(r, k-1)$. Each vertex of $B(r, k)$ corresponding to an arc $v w$ of $\vec{B}(r, k-1)$ is in exactly two of these sets, namely $B_{v}$ and $B_{w}$. The endpoints of each edge of $B(r, k)$ corresponding to a path $u v, v w$ of $\vec{B}(r, k-1)$ are both in $B_{v}$. These $r^{k-1}$ sets, one for each vertex of $\vec{B}(r, k-1)$, define the desired sets in $B(r, k)$.

The next two lemmas will be useful later.

Lemma 2. For every integer $q>1$ there is a $(2 q-2)$-regular graph $L$ with $\left(\begin{array}{c}q+1 \\ 2\end{array}\right)$ vertices, containing cliques $L_{1}, \ldots, L_{q+1}$ each of order $q$, such that each vertex in $L$ is in exactly two of the $L_{i}$, and $L_{i} \cap L_{j} \neq \emptyset$ for all $i, j \in[1, q+1]$.

Proof. Let $L$ be the line graph of the complete graph $K_{q+1}$. That is, $V(L):=\{\{i, j\}$ : $1 \leqslant i, j \leqslant q+1, i \neq j\}$, where $L_{i}:=\{\{i, j\}: 1 \leqslant j \leqslant q+1, i \neq j\}$ is a clique for each $i \in[1, q+1]$. The claimed properties are immediate. 
Lemma 3. For all integers $p \geqslant 1$ and $q \geqslant 1$ and $m \leqslant(q+1) p$ there is a bipartite graph $T$ with bipartition $C, D$, such that $C$ consists of $m$ vertices each with degree $q$, and $D$ consists of $\left(\begin{array}{c}q+1 \\ 2\end{array}\right)$ vertices each with degree at most $2 p$, and every pair of vertices in $C$ have a common neighbour in $D$.

Proof. By Lemma 2, there is a set $D=V(L)$ of size $\left(\begin{array}{c}q+1 \\ 2\end{array}\right)$, containing subsets $D_{1}, \ldots, D_{q+1}$ each of size $q$, such that each element of $D$ is in exactly two of the $D_{i}$, and $D_{i} \cap D_{j} \neq \emptyset$ for all $i, j \in[1, q+1]$.

Let $T$ be the graph with vertex set $C \cup D$, where $C$ is defined as follows. For each $i \in[1, q+1]$ add a set $C_{i}$ of $p$ vertices to $C$, each adjacent to every vertex in $D_{i}$. Since $\left|D_{i}\right|=q$, each vertex in $C$ has degree $q$. Since each element of $D$ is in exactly two of the $D_{i}$, each vertex in $D$ has degree $2 p$.

Consider two vertices $v, w \in C$. Say $v \in C_{i}$ and $w \in C_{j}$. Let $x$ be a vertex in $D_{i} \cap D_{j}$. Then $x$ is a common neighbour of $v$ and $w$ in $G$.

We have proved that $T$ has the desired properties in the case that $m=(q+1) p$. Finally, delete $(q+1) p-m$ vertices from $C$, and the obtained graph has the desired properties.

\section{Average Degree}

This section presents bounds on the maximum number of vertices in a graph with given average degree. For fixed diameter, the upper and lower bounds are within a constant factor. We have the following rough upper bound for graphs of given minimum degree.

Proposition 4. Every graph with minimum degree $\delta$, maximum degree $\Delta$ and diameter $k$ has at most $2 \delta(\Delta-1)^{k-1}+1$ vertices.

Proof. Let $v$ be a vertex of degree $\delta$. For $0 \leqslant i \leqslant k$, let $n_{i}$ be the number of vertices at distance $i$ from $v$. Thus $n_{0}=1$ and $n_{i} \leqslant \delta(\Delta-1)^{i-1}$ for all $i \geqslant 1$. In total, $n=\sum_{i=0}^{k} n_{i} \leqslant 1+\sum_{i=1}^{k} \delta(\Delta-1)^{i-1}=1+\delta \frac{(\Delta-1)^{k}-1}{\Delta-2} \leqslant 1+2 \delta(\Delta-1)^{k-1}$.

Since minimum degree is at most average degree, we have the following corollary.

Corollary 5. Every graph with average degree d, maximum degree $\Delta$ and diameter $k$ has at most $2 d(\Delta-1)^{k-1}+1$ vertices.

The following is the main result of this section; it says that Corollary 5 is within a constant factor of optimal for fixed $k$.

Proposition 6. For all integers $d \geqslant 4$ and $k \geqslant 3$ and $\Delta \geqslant 2 d$ there is a graph with average degree at most $d$, maximum degree at most $\Delta$, diameter at most $k$, and at least $\frac{d}{8}\left\lfloor\frac{\Delta}{4}\right\rfloor^{k-1}$ vertices.

Proof. Let $r:=\left\lfloor\frac{\Delta}{4}\right\rfloor$. Let $q:=\left\lfloor\frac{d}{4}\right\rfloor \geqslant 2$. Let $p:=\left\lfloor\frac{\Delta}{2}\right\rfloor-r-q+1$. Note that $d \geqslant 4 q$ and $4 p \geqslant \Delta-4 q \geqslant \frac{\Delta}{2}$. 
Let $B:=B(r, k-2)$ be the graph from Lemma 1 with maximum degree at most $2 r$, diameter $k-2$, and $r^{k-2}$ vertices.

Let $L$ be the $(2 q-2)$-regular graph from Lemma 2 with $\left(\begin{array}{c}q+1 \\ 2\end{array}\right)$ vertices, containing cliques $L_{1}, \ldots, L_{q+1}$ each of order $q$, such that each vertex in $L$ is in exactly two of the $L_{i}$, and $L_{i} \cap L_{j} \neq \emptyset$ for all $i, j \in[1, q+1]$.

Let $H$ be the cartesian product graph $L \square B$. Note that $H$ has $\left(\begin{array}{c}q+1 \\ 2\end{array}\right) r^{k-2}$ vertices and has maximum degree at most $2 q-2+2 r$. For $i \in[1, q+1]$ and $v \in V(B)$, let $X_{i, v}$ be the clique $\left\{(x, v): x \in L_{i}\right\}$ in $H$. Since each vertex in $L$ is in exactly two of the $L_{i}$, each vertex in $H$ is in exactly two of the $X_{i, v}$.

Let $G$ be the graph obtained from $H$ as follows: for $i \in[1, q+1]$ and $v \in V(B)$, add an independent set $Y_{i, v}$ of $p$ vertices to $G$ completely adjacent to $X_{i, v}$; that is, every vertex in $Y_{i, v}$ is adjacent to every vertex in $X_{i, v}$. We now prove that $G$ has the claimed properties.

The number of vertices in $G$ is

$$
|V(G)| \geqslant \sum_{i, v}\left|Y_{i, v}\right|=(q+1) r^{k-2} p \geqslant \frac{d}{4}\left\lfloor\frac{\Delta}{4}\right\rfloor^{k-2} \frac{\Delta}{8} \geqslant \frac{d}{8}\left\lfloor\frac{\Delta}{4}\right\rfloor^{k-1} .
$$

To determine the diameter of $G$, let $\alpha$ and $\beta$ be vertices in $G$. Say $\alpha \in X_{i, v} \cup Y_{i, v}$ and $\beta \in X_{j, w} \cup Y_{j, w}$. Let $x$ be a vertex in $L_{i} \cap L_{j}$. Let $v=y_{1}, \ldots, y_{\ell}=w$ be a path of length at most $k-2$ in $B$. Then $\alpha,\left(x, y_{1}\right),\left(x, y_{2}\right), \ldots,\left(x, y_{\ell}\right), \beta$ is path of length at most $k$ in $G$. Hence $G$ has diameter at most $k$.

Consider the maximum degree of $G$. Each vertex in some set $Y_{i, v}$ has degree $\left|X_{i, v}\right|=$ $\left|L_{i}\right|=q \leqslant \Delta$. Each vertex in some set $X_{i, v}$ has degree $2 q-2+2 r+2 p \leqslant \Delta$. Thus $G$ has maximum degree at most $\Delta$.

It remains to prove that the average degree of $G$ is at most $d$. There are $|V(H)|=$ $\left(\begin{array}{c}q+1 \\ 2\end{array}\right) r^{k-2}$ vertices of degree at most $\Delta$, and there are $(q+1) r^{k-2} p$ vertices of degree $q$. Thus the average degree is at most

$$
\frac{\left(\begin{array}{c}
q+1 \\
2
\end{array}\right) r^{k-2} \cdot \Delta+(q+1) r^{k-2} p q}{\left(\begin{array}{c}
q+1 \\
2
\end{array}\right) r^{k-2}+(q+1) r^{k-2} p}=\frac{\frac{q}{2} \Delta+p q}{\frac{q}{2}+p}
$$

Hence it suffices to prove that $q \Delta+2 p q \leqslant(q+2 p) d$. Since $\Delta \geqslant 2 d$ and $d \geqslant 4 q$,

$$
d \Delta=\frac{d \Delta}{2}+\frac{d \Delta}{2} \geqslant \frac{d \Delta}{2}+d^{2} \geqslant 2 q \Delta+4 d q .
$$

That is, $2 d \Delta-2 q \Delta-8 q d \geqslant d \Delta-4 q d$. Since $4 p \geqslant \Delta-4 q$ and $8 q^{2} \geqslant 0$,

$$
8 p(d-q) \geqslant 2(\Delta-4 q)(d-q)=2 d \Delta-2 q \Delta-8 q d+8 q^{2} \geqslant d \Delta-4 q d \geqslant 4 q \Delta-4 q d .
$$

That is, $4 p d+2 q d \geqslant 2 q \Delta+4 p q$, as desired. Hence the average degree of $G$ is at most $d$.

Note that for particular values of $k$ and $\Delta$, other graphs can be used instead of the de Bruijn graph in the proof of Proposition 6 to improve the constants in our results; we omit all these details. 


\section{Arboricity}

This section proves that the maximum number of vertices in a graph with arboricity $b$ is $f(b, k) \cdot \Delta^{\lfloor k / 2\rfloor}$ for some function $f$. Reasonably tight lower and upper bounds on $f$ are established. First we prove the upper bound.

Theorem 7. For every graph $G$ with arboricity $b$, diameter $k$, and maximum degree $\Delta$,

$$
|V(G)| \leqslant 4 k(2 b)^{k} \Delta^{\lfloor k / 2\rfloor}+1 .
$$

Proof. Let $G_{1}, \ldots, G_{b}$ be spanning forests of $G$ whose union is $G$. Orient the edges of each component of each $G_{i}$ towards a root vertex. The choice of the root is arbitrary. Thus each vertex $v$ of $G$ has outdegree at most 1 in each $G_{i}$; therefore $v$ has outdegree at most $b$ in $G$.

Consider an unordered pair of vertices $\{v, w\}$. Let $P$ be a shortest $v w$-path in $G$. Say $P$ has $\ell$ edges. Then $\ell \leqslant k$. An edge of $P$ oriented in the direction from $v$ to $w$ is called forward. If at least $\left\lceil\frac{\ell}{2}\right\rceil$ of the edges in $P$ are forward, then charge the pair $\{v, w\}$ to $v$, otherwise charge $\{v, w\}$ to $w$.

Consider a vertex $v$. If some pair $\{v, w\}$ is charged to $v$ then there is path of length $\ell$ from $v$ to $w$ with exactly $i$ forward arcs, for some $i$ and $\ell$ with $\left\lceil\frac{\ell}{2}\right\rceil \leqslant i \leqslant \ell \leqslant k$. Since each vertex has outdegree at most $b$, the number of such paths is at most $\left(\begin{array}{l}\ell \\ i\end{array}\right) b^{i} \Delta^{\ell-i}$. Hence the number of pairs charged to $v$ is at most

$$
\begin{aligned}
\sum_{\ell=1}^{k} \sum_{i=\lceil\ell / 2\rceil}^{\ell}\left(\begin{array}{l}
\ell \\
i
\end{array}\right) b^{i} \Delta^{\ell-i} & \leqslant k \sum_{i=\lceil k / 2\rceil}^{k}\left(\begin{array}{l}
k \\
i
\end{array}\right) b^{i} \Delta^{k-i} \\
& =k \sum_{i=0}^{\lfloor k / 2\rfloor}\left(\begin{array}{c}
k \\
k-i
\end{array}\right) b^{k-i} \Delta^{i} \\
& \leqslant k 2^{k} b^{k} \sum_{i=0}^{\lfloor k / 2\rfloor} \Delta^{i} \\
& \leqslant 2 k(2 b)^{k} \Delta^{\lfloor k / 2\rfloor} .
\end{aligned}
$$

Hence, the total number of pairs, $\left(\begin{array}{l}n \\ 2\end{array}\right)$, is at most $2 k(2 b)^{k} \Delta^{\lfloor k / 2\rfloor} n$. The result follows.

We now show that the upper bound in Theorem 7 is close to being best possible (for fixed $k)$.

Theorem 8. For all even integers $b \geqslant 2$ and $k \geqslant 4$ and $\Delta \geqslant b$, such that $\Delta \equiv 2(\bmod 4)$ or $b \equiv 0(\bmod 4)$, there is a graph $G$ with arboricity at most $b$, maximum degree at most $\Delta$, diameter at most $k$, and at least $\frac{8}{b^{2}}\left(\frac{b \Delta}{8}\right)^{k / 2}$ vertices.

Proof. Let $q:=\frac{\Delta}{2}$ and $p:=\frac{b}{2}$ and $\ell:=\frac{k}{2}-1$. Then $q, p$ and $\ell$ are positive integers. Let $r:=\frac{(q+1) p}{2}$. Then $r$ is a positive integer $($ since $\Delta \equiv 2(\bmod 4)$ or $b \equiv 0(\bmod 4))$. 
Let $B$ be the de Bruijn graph $B(r, \ell)$. By Lemma $1, B$ has diameter $\ell$ and $r^{\ell}$ vertices. Moreover, there are sets $B_{1}, \ldots, B_{r^{\ell-1}}$ of vertices in $B$, each containing $2 r-2$ or $2 r$ vertices, such that each vertex of $B$ is in exactly two of the $B_{i}$, and the endpoints of each edge in $B$ are in some $B_{i}$. Let $r_{i}:=\left|B_{i}\right|$. Thus $r_{i} \leqslant 2 r=(q+1) p$.

By Lemma 3 , for each $i \in\left[1, r^{\ell-1}\right]$ there is a bipartite graph $T_{i}$ with bipartition $B_{i}, D_{i}$, such that $B_{i}$ consists of $r_{i}$ vertices each with degree $q$, and $D_{i}$ consists of $\left(\begin{array}{c}q+1 \\ 2\end{array}\right)$ vertices each with degree at most $2 p \leqslant b$, and each pair of vertices in $B_{i}$ have a common neighbour in $D_{i}$.

Let $G$ be the bipartite graph with bipartition $V(B) \cup D$, where $D:=\cup_{i} D_{i}$ and the induced subgraph $G\left[B_{i}, D_{i}\right]$ is $T_{i}$. In $G$, each vertex in $V(B)$ has degree $2 q \leqslant \Delta$, and each vertex in $D$ has degree at most $b \leqslant \Delta$. Thus $G$ has maximum degree $\Delta$. Assign each edge in $G$ one of $b$ colours, such that two edges receive distinct colours whenever they have an endpoint in $D$ in common. Each colour class induces a subgraph in which each component is a star. Hence $G$ has arboricity at most $b$. Observe that

$$
|V(G)| \geqslant|D|=r^{\ell-1}\left(\begin{array}{c}
q+1 \\
2
\end{array}\right) \geqslant\left(\frac{b \Delta}{8}\right)^{\ell-1} \frac{\Delta^{2}}{8}=\left(\frac{b \Delta}{8}\right)^{k / 2-2} \frac{\Delta^{2}}{8}=\frac{8}{b^{2}}\left(\frac{b \Delta}{8}\right)^{k / 2} .
$$

It remains to prove that $G$ has diameter at most $k$. Consider two vertices $v$ and $w$ in $G$. If $v \in D_{i}$ then let $v^{\prime}$ be a neighbour of $v$ in $B_{i}$. If $v \in B_{i}$ then let $v^{\prime}$ be $v$. If $w \in D_{j}$ then let $w^{\prime}$ be a neighbour of $w$ in $B_{j}$. If $w \in B_{j}$ then let $w^{\prime}$ be $w$. In $B$, there is a $v^{\prime} w^{\prime}$-path $P$ of length at most $\ell$. For each edge $x y$ in $P$, both $x$ and $y$ are in some set $B_{a}$ (see Lemma 1). Since $x$ and $y$ have a common neighbour in $T_{a}$ (by Lemma 3), we can replace $x y$ in $P$ by a 2 -edge path in $T_{a}$, to obtain a $v^{\prime} w^{\prime}$-path in $G$ of length at most $2 \ell$. Possibly adding the edges $v v^{\prime}$ or $w w^{\prime}$ gives a $v w$-path in $G$ of length at most $2 \ell+2=k$. Hence $G$ has diameter at most $k$.

Consider the case of diameter 2 graphs with arboricity $b$. Every such graph has average degree less than $2 b$, and thus has at most $4 b \Delta$ vertices by Corollary 5 . We now show that this upper bound is within a constant factor of optimal. (This result is not covered by Theorem 8 which assumes $k \geqslant 4$.)

Proposition 9. For all integers $b \geqslant 1$ and even $\Delta \geqslant 4 b$ there is a graph with diameter 2 , arboricity at most $b$, maximum degree $\Delta$, and at least $\frac{b \Delta}{4}$ vertices.

Proof. By Lemma 2, there is a $(2 b-2)$-regular graph $X$ with $\left(\begin{array}{c}b+1 \\ 2\end{array}\right)$ vertices, containing cliques $X_{1}, \ldots, X_{b+1}$ each of order $b$, such that each vertex in $X$ is in exactly two of the $X_{i}$, and $X_{i} \cap X_{j} \neq \emptyset$ for all $i, j \in[1, q+1]$.

Initialise a graph $G$ equal to $X$. For $i \in[1, b+1]$, add an independent set $Y_{i}$ of $p:=\frac{\Delta}{2}-b+1$ vertices to $G$ completely adjacent to $X_{i}$.

Consider two vertices $v$ and $w$ in $G$. Say $v \in X_{i} \cup Y_{i}$ and $w \in X_{j} \cup Y_{j}$. Let $x$ be the vertex in $X_{i} \cap X_{j}$. If $v=x$ or $x=w$ then $v w$ is an edge in $G$, otherwise $v x w$ is a path in $G$. Thus $G$ has diameter 2 .

Vertices in each $X_{i}$ have degree $2 b-2+2 p=\Delta$ and vertices in each $Y_{i}$ have degree $b \leqslant \Delta$. Hence $G$ has maximum degree $\Delta$. The number of vertices in $G$ is more than $(b+1) p=(b+1)\left(\frac{\Delta}{2}-b+1\right) \geqslant \frac{b \Delta}{4}$. 
To calculate the arboricity of $G$, consider a subgraph $H$ of $G$. Let $x_{i}:=\left|X_{i} \cap V(H)\right|$ and $y_{i}:=\left|Y_{i} \cap V(H)\right|$. Since $x_{i} \leqslant\left|X_{i}\right|=b$ and $b \geqslant 2$,

$$
\sum_{i}\left(\begin{array}{c}
x_{i} \\
2
\end{array}\right)=\sum_{i} \frac{x_{i}\left(x_{i}-1\right)}{2} \leqslant \sum_{i} \frac{b\left(x_{i}-1\right)}{2}=\sum_{i} \frac{b x_{i}-b}{2}<\left(\sum_{i} \frac{b x_{i}}{2}\right)-b .
$$

Since $x_{i} y_{i} \leqslant\left|X_{i}\right| y_{i}=b y_{i}$,

$$
\sum_{i}\left(\begin{array}{c}
x_{i} \\
2
\end{array}\right)+x_{i} y_{i} \leqslant\left(\sum_{i} \frac{b x_{i}}{2}+b y_{i}\right)-b=b\left(\left(\sum_{i} \frac{x_{i}}{2}+y_{i}\right)-1\right) .
$$

Observe that $|E(H)| \leqslant \sum_{i}\left(\begin{array}{c}x_{i} \\ 2\end{array}\right)+x_{i} y_{i}$ and $|V(H)| \geqslant \sum_{i} \frac{x_{i}}{2}+y_{i}$ (since each vertex in $X$ is in exactly two of the $\left.X_{i}\right)$. Thus $|E(H)| \leqslant b(|V(H)|-1)$, and $G$ has arboricity at most $b$ by $(2)$.

We conclude this section with an open problem about the degree-diameter problem for graphs containing no $K_{t}$-minor. Every such graph has arboricity at most $c t \sqrt{\log t}$, for some constant $c>0$; see $[21,28,29]$. Thus Theorem 7 implies that for every $K_{t}$-minor-free graph $G$ with diameter $k$ and maximum degree $\Delta \gg t$,

$$
|V(G)| \leqslant 4 k(c t \sqrt{\log t})^{k} \Delta^{\lfloor k / 2\rfloor} .
$$

Improving the $f(t, k)$ term in this $f(t, k) \Delta^{\lfloor k / 2\rfloor}$ bound is a challenging open problem.

\section{Separators and Treewidth}

This section studies a separator-based approach for proving upper bounds in the degreediameter problem. A separation of order $s$ in an $n$-vertex graph $G$ is a partition $(A, S, B)$ of $V(G)$, such that $|A| \leqslant \frac{2}{3} n$ and $|B| \leqslant \frac{2}{3} n$ and $|S| \leqslant s$ and there is no edge between $A$ and $B$. Fellows et al. [12] first used separators to prove upper bounds in the degree-diameter problem. In particular, they implicitly proved that every graph that has a separation of order $s$ has $3 s M\left(\Delta,\left\lfloor\frac{k}{2}\right\rfloor\right)$ vertices. The following lemma improves the dependence on $s$ in this result when $k$ is even. We include the proof by Fellows et al. [12] for completeness.

Lemma 10. Let $G$ be a graph with maximum degree at most $\Delta$, and diameter at most $k$. Assume $(A, S, B)$ is a separation of order $s$ in $G$. Then

$$
|V(G)| \leqslant \begin{cases}3 s M\left(\Delta, \frac{k-1}{2}\right) & \text { if } k \text { is odd } \\ \frac{3}{2} \sqrt{s} \Delta(\Delta-1)^{k / 2-1}+3 s M\left(\Delta, \frac{k}{2}-1\right) & \text { if } k \text { is even } .\end{cases}
$$

Proof. Let $n:=|V(G)|$. Note that $|A| \geqslant n-|B|-s \geqslant \frac{n}{3}-s$. By symmetry, $|B| \geqslant \frac{n}{3}-s$. We use this fact repeatedly.

For $v \in A \cup B$, let $\operatorname{dist}(v, S):=\min \{\operatorname{dist}(v, x): x \in S\}$. If $\operatorname{dist}(v, S) \geqslant\lfloor k / 2\rfloor+1$ for some $v \in A$ and $\operatorname{dist}(w, S) \geqslant\lfloor k / 2\rfloor+1$ for some $w \in B$, then $\operatorname{dist}(v, w) \geqslant 2\lfloor k / 2\rfloor+2 \geqslant$ $k+1$, which is a contradiction. Hence, without loss of generality, $\operatorname{dist}(v, S) \leqslant\lfloor k / 2\rfloor$ for 
each $v \in A$. By the Moore bound, for each vertex $x \in S$, there are at most $M(\Delta,\lfloor k / 2\rfloor)-1$ vertices in $A$ at distance at most $\lfloor k / 2\rfloor$ from $x$. Each vertex in $A$ is thus counted. Hence

$$
\frac{n}{3}-s \leqslant|A| \leqslant s M(\Delta,\lfloor k / 2\rfloor)-s,
$$

implying $n \leqslant 3 s M(\Delta,\lfloor k / 2\rfloor)$. This proves the result of Fellows et al. [12] mentioned above, and proves the case of odd $k$ in the theorem.

Now assume that $k=2 \ell$ is even. Suppose on the contrary that

$$
\frac{n}{3}>\frac{\sqrt{s}}{2} \Delta(\Delta-1)^{\ell-1}+s M(\Delta, \ell-1) .
$$

First consider the case in which some vertex in $A$ is at distance at least $\ell+1$ from $S$. Thus every vertex in $B$ is at distance at most $\ell-1$ from $S$. By the Moore bound,

$$
s M(\Delta, \ell-1)-s \geqslant|B| \geqslant \frac{n}{3}-s>\frac{\sqrt{s}}{2} M(\Delta, \ell)+s M(\Delta, \ell-1)-s,
$$

which is a contradiction. Now assume that every vertex in $A$ is at distance at most $\ell$ from $S$. By symmetry, every vertex in $B$ is at distance at most $\ell$ from $S$.

Let $A^{\prime}$ and $B^{\prime}$ be the subsets of $A$ and $B$ respectively at distance exactly $\ell$ from $S$. By the Moore bound, $\left|A-A^{\prime}\right| \leqslant s M(\Delta, \ell-1)-s$. Hence

$$
\left|A^{\prime}\right|=|A|-\left|A-A^{\prime}\right| \geqslant \frac{n}{3}-s-s M(\Delta, \ell-1)+s>\frac{\sqrt{s}}{2} \Delta(\Delta-1)^{\ell-1} .
$$

By symmetry, $\left|B^{\prime}\right|>\frac{\sqrt{s}}{2} \Delta(\Delta-1)^{\ell-1}$.

Let $P:=\left\{(x, y): x \in A^{\prime}, y \in B^{\prime}\right\}$. For each pair $(x, y) \in P$, some vertex $v$ in $S$ is at distance $\ell$ from both $x$ and $y$. Charge $(x, y)$ to $v$. We now bound the number of pairs in $P$ charged to each vertex $v \in S$. Say $v$ has degree $a$ in $A$ and degree $b$ in $B$. Thus $a+b \leqslant \Delta$. There are at most $a(\Delta-1)^{\ell-1}$ vertices at distance exactly $\ell$ from $v$ in $A$, and there at most $b(\Delta-1)^{\ell-1}$ vertices at distance exactly $\ell$ from $v$ in $B$. Thus the number of pairs charged to $v$ is at most

$$
a b(\Delta-1)^{2 \ell-2} \leqslant \frac{1}{4}(a+b)^{2}(\Delta-1)^{2 \ell-2} \leqslant \frac{1}{4} \Delta^{2}(\Delta-1)^{2 \ell-2} .
$$

Hence

$$
\frac{s}{4} \Delta^{2}(\Delta-1)^{2 \ell-2}=\left(\frac{\sqrt{s}}{2} \Delta(\Delta-1)^{\ell-1}\right)^{2}<\left|A^{\prime}\right| \cdot\left|B^{\prime}\right|=|P| \leqslant \frac{s}{4} \Delta^{2}(\Delta-1)^{2 \ell-2} .
$$

This contradiction proves that $n \leqslant \frac{3}{2} \sqrt{s} \Delta(\Delta-1)^{\ell-1}+3 s M(\Delta, \ell-1)$.

Lemma 10 can be written in the following convenient form.

Lemma 11. For all $\epsilon>0$ there is a constant $c_{\epsilon}$ such that for every graph $G$ with maximum degree $\Delta$, diameter $k$, and a separation of order $s$,

$$
|V(G)| \leqslant \begin{cases}(3+\epsilon) s(\Delta-1)^{(k-1) / 2} & \text { if } k \text { is odd and } \Delta \geqslant c_{\epsilon} \\ \left(\frac{3}{2}+\epsilon\right) \sqrt{s}(\Delta-1)^{k / 2} & \text { if } k \text { is even and } \Delta \geqslant c_{\epsilon} \sqrt{s} .\end{cases}
$$


Proof. First consider the the odd $k$ case. For $\Delta \geqslant \frac{6}{\epsilon}+2$ we have $3\left(\frac{\Delta}{\Delta-2}\right) \leqslant 3+\epsilon$. Thus, by Lemma 10 and the Moore bound,

$$
|V(G)| \leqslant 3 s\left(\frac{\Delta}{\Delta-2}\right)(\Delta-1)^{(k-1) / 2} \leqslant(3+\epsilon) s(\Delta-1)^{(k-1) / 2} .
$$

Now consider the even $k$ case. For $\Delta \geqslant \frac{3}{\epsilon}+1$ we have $\frac{3}{2} \Delta \leqslant\left(\frac{3}{2}+\frac{\epsilon}{2}\right)(\Delta-1)$. And for $\Delta \geqslant \frac{9}{\epsilon} \sqrt{s}+2$ we have $3 \sqrt{s} \leqslant \frac{\epsilon}{3}(\Delta-2) \leqslant \frac{\epsilon}{2}\left(\frac{\Delta-1}{\Delta}\right)(\Delta-2)$, implying $3 s\left(\frac{\Delta}{\Delta-2}\right) \leqslant \frac{\epsilon}{2} \sqrt{s}(\Delta-1)$. Hence, by Lemma 10 and the Moore bound,

$$
\begin{aligned}
|V(G)| & \leqslant \frac{3}{2} \sqrt{s} \Delta(\Delta-1)^{k / 2-1}+3 s\left(\frac{\Delta}{\Delta-2}\right)(\Delta-1)^{k / 2-1} \\
& \leqslant\left(\frac{3}{2}+\frac{\epsilon}{2}\right) \sqrt{s}(\Delta-1)^{k / 2}+\frac{\epsilon}{2} \sqrt{s}(\Delta-1)^{k / 2} \\
& \leqslant\left(\frac{3}{2}+\epsilon\right) \sqrt{s}(\Delta-1)^{k / 2} .
\end{aligned}
$$

This completes the proof of the lemma.

Treewidth is a key topic when studying separators. In particular, every graph with treewidth $t$ has a separation of order $t+1$, and in fact, a converse result holds [26]. Thus Lemma 11 implies:

Theorem 12. For all $\epsilon>0$ there is a constant $c_{\epsilon}$ such that for every graph $G$ with maximum degree $\Delta$, treewidth $t$, and diameter $k$,

$$
|V(G)| \leqslant \begin{cases}(3+\epsilon)(t+1)(\Delta-1)^{(k-1) / 2} & \text { if } k \text { is odd and } \Delta \geqslant c_{\epsilon} \\ \left(\frac{3}{2}+\epsilon\right) \sqrt{t+1}(\Delta-1)^{k / 2} & \text { if } k \text { is even and } \Delta \geqslant c_{\epsilon} \sqrt{t+1}\end{cases}
$$

Note that Theorem 12 in the case of odd $k$ can also be concluded from a result by Gavoille et al. [15, Theorem 3.2]. Our original contribution is for the even $k$ case. We now show that both upper bounds in Theorem 12 are within a constant factor of optimal.

Proposition 13. For all integers $k \geqslant 1$ and $t \geqslant 2$ and $\Delta$ there is a graph $G$ with maximum degree $\Delta$, diameter $k$, treewidth at most $t$, and

$$
|V(G)| \geqslant \begin{cases}\frac{1}{2}(t+1)(\Delta-1)^{(k-1) / 2} & \text { if } k \text { is odd and } \Delta \geqslant 2 t-2 \\ \frac{1}{2} \sqrt{t+1}(\Delta-1)^{k / 2} & \text { if } k \text { is even and } \Delta \geqslant 4 \sqrt{2 t}\end{cases}
$$

Proof. First consider the case of odd $k$. Let $T$ be the rooted tree such that the root vertex has degree $\Delta-t$, every non-root non-leaf vertex has degree $\Delta$, and the distance between the root and each leaf equals $\frac{k-1}{2}$. Since $t \geqslant 2$ and $\frac{\Delta-t}{\Delta-2} \geqslant \frac{1}{2}$,

$$
\begin{aligned}
|V(T)|=1+(\Delta-t) \sum_{i=0}^{(k-3) / 2}(\Delta-1)^{i} & =\frac{t-2+(\Delta-t)(\Delta-1)^{(k-1) / 2}}{\Delta-2} \\
& \geqslant \frac{1}{2}(\Delta-1)^{(k-1) / 2} .
\end{aligned}
$$


Take $t+1$ disjoint copies of $T$, and add a clique on their roots. This graph is chordal with maximum clique size $t+1$. Thus it has treewidth $t$. The maximum degree is $\Delta$ and the number of vertices is at least $\frac{1}{2}(t+1)(\Delta-1)^{(k-1) / 2}$.

Now consider the case of even $k$. Let $q$ be the maximum integer such that $\left(\begin{array}{c}q+1 \\ 2\end{array}\right) \leqslant t+1$. Thus $2 \leqslant q \leqslant \sqrt{2 t} \leqslant \frac{\Delta}{4}$ and $q+1 \geqslant \sqrt{t+1}$. Let $T$ be the tree, rooted at $r$, such that $r$ has degree $\Delta-q$, every non-leaf non-root vertex has degree $\Delta$, and the distance between $r$ and each leaf is $\frac{k}{2}-1$. Since $q \geqslant 2$ and $\frac{\Delta-q}{\Delta-2} \geqslant \frac{1}{2}$,

$$
\begin{aligned}
|V(T)|=1+(\Delta-q) \sum_{i=0}^{k / 2-2}(\Delta-1)^{i} & =\frac{q-2+(\Delta-q)(\Delta-1)^{k / 2-1}}{\Delta-2} \\
& \geqslant \frac{1}{2}(\Delta-1)^{k / 2-1} .
\end{aligned}
$$

By Lemma 2 , there is a $(2 q-2)$-regular graph $L$ with $\left(\begin{array}{c}q+1 \\ 2\end{array}\right)$ vertices, containing cliques $L_{1}, \ldots, L_{q+1}$ each of order $q$, such that each vertex in $L$ is in exactly two of the $L_{i}$, and $L_{i} \cap L_{j} \neq \emptyset$ for all $i, j \in[1, q+1]$. Let $G$ be the graph obtained from $L$ as follows. For each $i \in[1, q+1]$, add $\Delta-2(q-1)$ disjoint copies of $T$ (called $i$-copies), where every vertex in $L_{i}$ is adjacent to the roots of the $i$-copies of $T$, as illustrated in Figure 1 . It is easily verified that $G$ has maximum degree $\Delta$. Consider a vertex $v$ in some $i$-copy of $T$ or in $L_{i}$, and a vertex $w$ in some $j$-copy of $T$ or in $L_{j}$. Let $x$ be in $L_{i} \cap L_{j}$. Then $\operatorname{dist}(v, x) \leqslant \frac{k}{2}$ and $\operatorname{dist}(w, x) \leqslant \frac{k}{2}$, implying $\operatorname{dist}(v, w) \leqslant k$. Hence $G$ has diameter at most $k$. Let $G^{\prime}$ be the supergraph of $G$ obtained by adding a clique on $V(L)$. Thus $G^{\prime}$ is chordal with maximum clique size $\left(\begin{array}{c}q+1 \\ 2\end{array}\right) \leqslant t+1$. Hence $G$ has treewidth at most $t$. The number of vertices in $G$ is at least $(q+1)(\Delta-2 q+2)|V(T)| \geqslant \sqrt{t+1} \cdot \frac{\Delta}{2} \cdot(\Delta-1)^{k / 2-1}$.

We now consider the degree-diameter problem for graphs with given Euler genus. Note that the case of planar graphs has been widely studied [12, 13, 19, 25, 30, 31]. Šiagiová and Simanjuntak [27] proved that for every graph $G$ with Euler genus $g$,

$$
|V(G)| \leqslant c(g+1) k(\Delta-1)^{\lfloor k / 2\rfloor},
$$

for some absolute constant $c$. Eppstein [11] proved that every graph with Euler genus $g$ and diameter $k$ has treewidth at most $c(g+1) k$ for some absolute constant $c$, and Dujmovic et al. [10] proved the explicit bound of $(2 g+3) k$. Theorem 12 thus implies the upper bound in (3) and improves upon it when $k$ is even:

Theorem 14. For all $\epsilon>0$ there is a constant $c_{\epsilon}$ such that for every graph $G$ with Euler genus $g$, maximum degree $\Delta$ and diameter $k$,

$$
|V(G)| \leqslant \begin{cases}(3+\epsilon)((2 g+3) k+1)(\Delta-1)^{(k-1) / 2} & \text { for odd } k \text { and } \Delta \geqslant c_{\epsilon} \\ \left(\frac{3}{2}+\epsilon\right) \sqrt{(2 g+3) k+1}(\Delta-1)^{k / 2} & \text { for even } k \text { and } \Delta \geqslant c_{\epsilon} \sqrt{(2 g+3) k+1}\end{cases}
$$

In our companion paper [25] we further investigate the degree-diameter problem for graphs on surfaces, providing an improved upper bound and a new lower bound. 


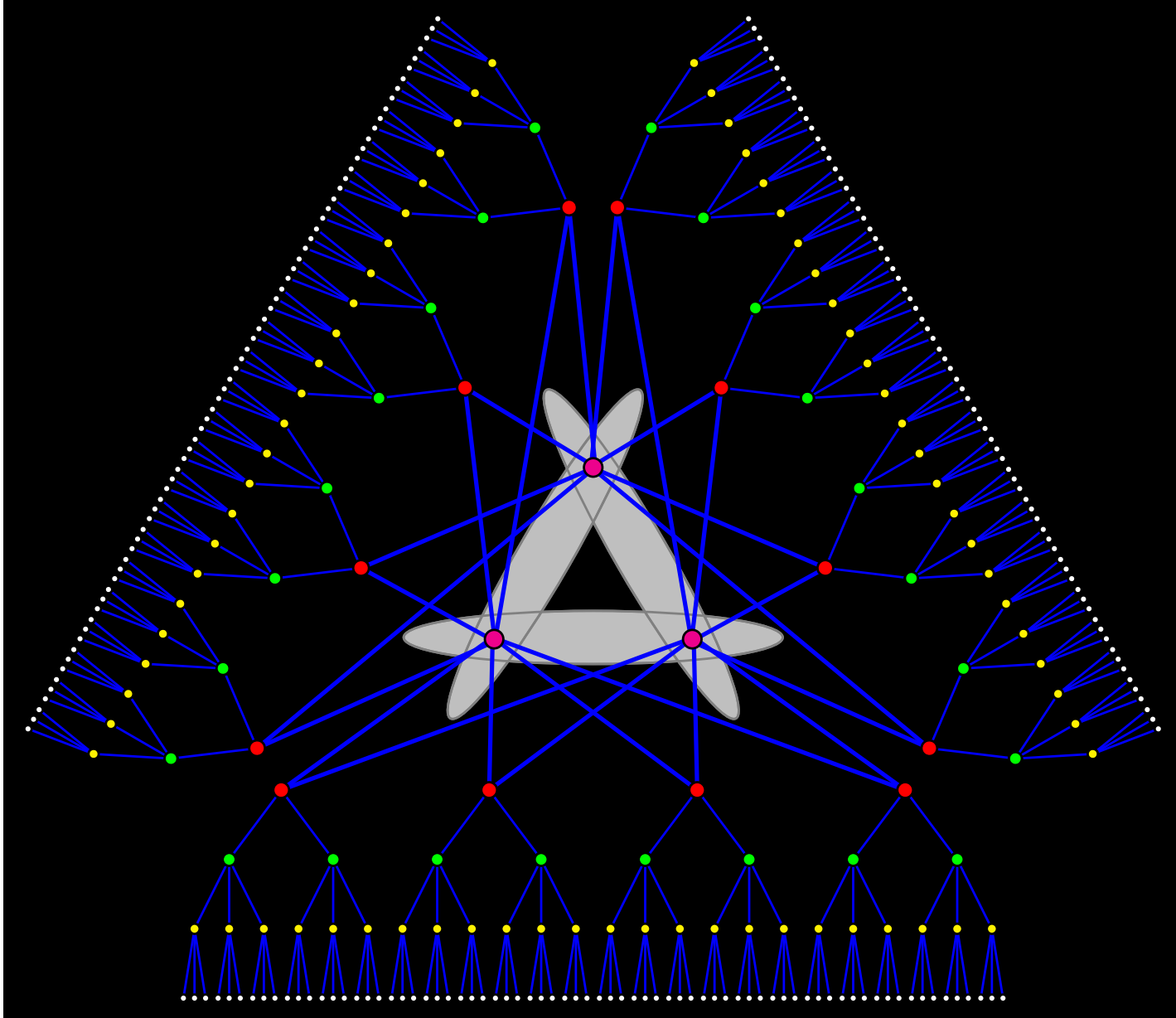

Figure 1: Construction in Proposition 13 for even $k$. Here $\Delta=4$ and $k=8$ and $t=2$.

To obtain an upper bound of the form $n \leqslant f(k) \Delta^{\lfloor k / 2\rfloor}$ using the separator-based approach, one needs a separation of order bounded by a function of the graph's diameter. In some sense, the graphs that have a separation of bounded order are precisely the graphs with bounded treewidth. See Reed's survey [26] for a precise statement here. Thus the separator-based method only works for graphs whose treewidth is bounded by a function of their diameter. The minor-closed graph classes with this property are precisely those that exclude a fixed apex graph as a minor [11]. Here a graph $H$ is apex if $H-v$ is planar for some vertex $v$ of $H$. For example, $K_{5}$ and $K_{3,3}$ are apex. Eppstein [11] proved that for some apex graph $H$ and some function $f$ (depending on $H$ ), the treewidth of every $H$-minor-free graph $G$ is at most $f(\operatorname{diam}(G))$. This is called the diameter-treewidth or bounded local treewidth property; also see [6, 7, 10, 17]. Demaine and Hajiaghayi [8] strengthened Eppstein's result by showing that one can take $f(k)=c k$ for some constant $c=c(H)$. Thus the next result follows from Theorem 12 . 
Theorem 15. For every fixed apex graph $H$ there is a constant $c=c(H)$, such that for every $H$-minor-free graph $G$ with diameter $k$,

$$
|V(G)| \leqslant \begin{cases}c k(\Delta-1)^{(k-1) / 2} & \text { if } k \text { is odd } \\ c \sqrt{k}(\Delta-1)^{k / 2} & \text { if } k \text { is even and } \Delta \geqslant c \sqrt{k} .\end{cases}
$$

As discussed above, for minor-closed classes, Theorem 15 is the strongest possible result that can be obtained using the separator-based method.

\section{3-Colourable and Triangle-Free Graphs}

As mentioned in the introduction, it is well known that the maximum number of vertices in a bipartite graph is $f(k) \Delta^{k-1}$. We now show that this bound does not hold for the more general class of 3-colourable graphs. In fact, we construct 3-colourable graphs where the number of vertices is within a constant factor of the Moore bound. First note that Kawai and Shibata [20] (building on the work of Harner and Entringer [18]) proved that for large $k \gtrsim \log r$, the de Bruijn graph $B(r, k)$, which roughly has $\left(\frac{\Delta}{2}\right)^{k}$ vertices, is 3 -colourable. The constructions below have the advantage of not assuming that $k$ is large.

In what follows a pseudograph is an undirected graph possibly with loops. A loop at a vertex $v$ counts for 1 in the degree of $v$. A pseudograph $H$ is $k$-good if for all (not necessarily distinct) vertices $v$ and $w$ there is a $v w$-walk of length exactly $k$ in $H$.

Given pseudographs $H_{1}$ and $H_{2}$, the direct product graph $H_{1} \times H_{2}$ has vertex set $V\left(H_{1}\right) \times V\left(H_{2}\right)$, where $(v, x)(w, y) \in E\left(H_{1} \times H_{2}\right)$ if and only if $v w \in E\left(H_{1}\right)$ and $x y \in$ $E\left(H_{2}\right)$.

Lemma 16. Let $H_{1}$ and $H_{2}$ be $k$-good pseudographs with maximum degree $\Delta_{1}$ and $\Delta_{2}$ respectively. Then $H_{1} \times H_{2}$ has $\left|V\left(H_{1}\right)\right| \cdot\left|V\left(H_{2}\right)\right|$ vertices, maximum degree $\Delta_{1} \Delta_{2}$, and diameter at most $k$. Moreover, if $\mathrm{H}_{2}$ is loopless and c-colourable, then $\mathrm{H}_{1} \times \mathrm{H}_{2}$ is ccolourable.

Proof. Clearly $H_{1} \times H_{2}$ has $\left|V\left(H_{1}\right)\right| \cdot\left|V\left(H_{2}\right)\right|$ vertices and maximum degree $\Delta_{1} \Delta_{2}$. Let $(v, x)$ and $(w, y)$ be distinct vertices of $G$. To prove that $G$ has diameter at most $k$, we construct a $(v, x)(w, y)$-walk of length at most $k$ in $G$. Since $H_{1}$ is $k$-good, there is a walk $v=v_{0}, v_{1}, \ldots, v_{k}=w$ of length $k$ in $H_{1}$. Since $H_{2}$ is $k$-good, there is a walk $x=$ $x_{0}, x_{1}, \ldots, x_{k}=y$ of length $k$ in $H_{2}$. Thus $(v, x)=\left(v_{0}, x_{0}\right),\left(v_{1}, x_{1}\right), \ldots,\left(v_{k}, x_{k}\right)=(w, y)$ is a walk of length $k$ between $(v, x)$ and $(w, y)$ in $H_{1} \times H_{2}$. Hence $H_{1} \times H_{2}$ has diameter at most $k$. Finally, colouring each vertex $(v, x)$ of $H_{1} \times H_{2}$ by the colour assigned to $x$ in a $c$-colouring of $\mathrm{H}_{2}$ gives a $c$-colouring of $\mathrm{H}_{1} \times \mathrm{H}_{2}$.

Lemma 17. $K_{3}$ is $k$-good for all $k \geqslant 2$.

Proof. Let $v, w \in V\left(K_{3}\right)=\{0,1,2\}$. If there is a $v w$-walk of length $k-2$, then there is a $v w$-walk of length $k$ (just repeat one edge twice). Thus the claim follows from the $k=2$ and $k=3$ cases. Without loss of generality, $v=0$. For $k=2$, one of 010,021 and 012 is a $v w$-walk of length 2 . For $k=3$, one of 0120,0121 and 0102 is a $v w$-walk of length 3 . 
To obtain results for triangle-free graphs we use the following:

Lemma 18. $C_{5}$ is $k$-good for all $k \geqslant 4$.

Proof. Say $V\left(C_{5}\right)=\{0,1,2,3,4\}$ and $E\left(C_{5}\right)=\{01,12,23,34,40\}$. Let $v, w \in V\left(C_{5}\right)$. If there is a $v w$-walk of length $k-2$, then there is a $v w$-walk of length $k$ (just repeat one edge twice). Thus the claim follows from the $k=4$ and $k=5$ cases. Without loss of generality, $v=0$. For $k=4$, one of 01010, 04321, 01212, 04343 and 01234 is a $v w$-walk of length 4 . For $k=5$, one of 012340, 040101, 043232, 012323 and 010404 is a $v w$-walk of length 5 .

Lemmas 16 and 17 imply:

Lemma 19. Let $H$ be a $k$-good pseudograph with maximum degree $\Delta$ for some $k \geqslant 2$. Then $H \times K_{3}$ is a 3-colourable graph with $3|V(H)|$ vertices, maximum degree $2 \Delta$, and diameter at most $k$.

Lemma 20. Let $H$ be a k-good pseudograph with maximum degree $\Delta$ for some $k \geqslant 4$. Then $H \times C_{5}$ is a 3-colourable triangle-free graph with $5|V(H)|$ vertices, maximum degree $2 \Delta$, and diameter at most $k$.

Proof. For any graph $G$ (without loops), if $H \times G$ contains a triangle $(a, u)(b, v)(c, w)$, then $u v w$ is a triangle in $G$ (even if $H$ has loops). Since $C_{5}$ is triangle-free, $H \times C_{5}$ is triangle-free. Thus Lemmas 16 and 18 imply the claim.

For particular values of $\Delta$ and $k$, various constructions for the degree-diameter problem can be used in the following lemma to give large 3-colourable and triangle-free graphs.

Proposition 21. Let $H$ be a graph with maximum degree $\Delta$ and diameter $k \geqslant 2$. Then there is a 3-colourable graph with $3|V(H)|$ vertices, maximum degree $2 \Delta+2$ and diameter at most $k$. Moreover, if $k \geqslant 4$ then there is a 3-colourable triangle-free graph with $5|V(H)|$ vertices, maximum degree $2 \Delta+2$, and diameter at most $k$.

Proof. Let $H^{\prime}$ be the pseudograph obtained from $H$ by adding a loop at each vertex. Thus $H^{\prime}$ is $k$-good and has maximum degree $\Delta+1$. Lemmas 19 and 20 imply that $H^{\prime} \times K_{3}$ and $H^{\prime} \times C_{5}$ satisfy the claims.

This result implies that for fixed $k \geqslant 2$ and $\Delta \gg k$, the maximum number of vertices in a 3-colourable graph is within a constant factor of the unrestricted case. And the same conclusion holds for $k \geqslant 4$ for 3-colourable triangle-free graphs.

We now give a concrete example:

Theorem 22. For all integers $\Delta \geqslant 4$ and $k \geqslant 2$, there is a 3-colourable graph with $3\left\lfloor\frac{\Delta}{4}\right\rfloor^{k}$ vertices, maximum degree at most $\Delta$, and diameter at most $k$. Moreover, if $k \geqslant 4$ then there is a 3-colourable triangle-free graph with $5\left\lfloor\frac{\Delta}{4}\right\rfloor^{k}$ vertices, maximum degree at most $\Delta$, and diameter at most $k$. 
Proof. Let $r:=\left\lfloor\frac{\Delta}{4}\right\rfloor$. Let $H$ be the undirected pseudograph underlying the de Bruijn digraph $\vec{B}(r, k)$ including any loops. Lemma 1 shows that $H$ has $r^{k}$ vertices, maximum degree at most $2 r$, and is $k$-good. Lemma 19 shows that $H \times K_{3}$ satisfies the first claim. Lemma 20 implies that $H \times C_{5}$ satisfies the second claim.

We now give ad-hoc constructions of triangle-free graphs with diameter 2 and 3 . These lower bounds are within a constant factor of the Moore bound. Let $\mathbb{Z}_{p}$ be the cyclic group with $p$ elements. For $a, b \in \mathbb{Z}_{p}$, let $\operatorname{dist}(a, b):=\min \{a-b, b-a\}$. Here, as always, addition and subtraction are in the group, so $\operatorname{dist}(a, b) \geqslant 0$.

Proposition 23. For all $\Delta \geqslant 20$ there is a triangle-free graph with diameter 2 , maximum degree at most $\Delta$, and at least $\left(2\left\lfloor\frac{\Delta+4}{8}\right\rfloor+2\right)^{2}$ vertices.

Proof. Let $p:=2\left\lfloor\frac{\Delta+4}{8}\right\rfloor+2$. Thus $p \geqslant 8$ is even. Let $G$ be a graph with vertex set $\mathbb{Z}_{p}^{2}$. Thus $|V(G)|=\left(2\left\lfloor\frac{\Delta+4}{8}\right\rfloor+2\right)^{2}$. Let $\left(v_{1}, v_{2}\right)$ denote a vertex $v$ in $G$. For distinct vertices $v$ and $w$, define the vw-vector to be $(a, b)$, where $a \leqslant b$ and $\{a, b\}=\left\{\operatorname{dist}\left(v_{1}, w_{1}\right)\right.$, $\left.\operatorname{dist}\left(v_{2}, w_{2}\right)\right\}$. Then $v w \in E(G)$ if and only if $a=1$ and $b \neq 2$. Observe that $G$ is $4(p-3)$-regular, and $4(p-3) \leqslant \Delta$.

We now show that the distance between distinct vertices $v, w$ in $G$ is at most 2 . Consider the following cases for the $v w$-vector $(a, b)$, where without loss of generality, $(a, b)=\left(\operatorname{dist}\left(v_{1}, w_{1}\right), \operatorname{dist}\left(v_{2}, w_{2}\right)\right)$ :

Case $(0, \geqslant 1)$ : Since $p \geqslant 8$, there exists $y \in \mathbb{Z}_{p}$ such that $\operatorname{dist}\left(v_{2}, y\right) \notin\{0,2\}$ and $\operatorname{dist}\left(w_{2}, y\right) \notin\{0,2\}$. Then $\left(v_{1}+1, y\right)=\left(w_{1}+1, y\right)$ is a common neighbour of $v$ and $w$.

Case $(1,2)$ : Since $p \geqslant 8$, there exists $x \in \mathbb{Z}_{p}$ such that $\operatorname{dist}\left(v_{1}, x\right) \notin\{0,2\}$ and $\operatorname{dist}\left(w_{1}, x\right) \notin\{0,2\}$. Since $\operatorname{dist}\left(v_{2}, w_{2}\right)=2$ there exists $y \in \mathbb{Z}_{p}$ such that $\operatorname{dist}\left(v_{2}, y\right)=$ $\operatorname{dist}\left(w_{2}, y\right)=1$. Then $(x, y)$ is a common neighbour of $v$ and $w$.

Case $(1, \neq 2)$ : Then $v$ and $w$ are adjacent.

Case $(\geqslant 2, \geqslant 2)$ : Since $p \geqslant 8$, there exists $x \in \mathbb{Z}_{p}$ such that $\operatorname{dist}\left(w_{1}, x\right)=1$ and $\operatorname{dist}\left(v_{1}, x\right) \notin\{0,2\}$. Similarly, there exists $y \in \mathbb{Z}_{p}$ such that $\operatorname{dist}\left(w_{2}, y\right) \notin\{0,2\}$ and $\operatorname{dist}\left(y, v_{1}\right)=1$. Then $(x, y)$ is a common neighbour of $v$ and $w$.

Suppose on the contrary that $G$ contains a triangle $T$. For each edge $u v$ of $T$, we have $\operatorname{dist}\left(u_{i}, v_{i}\right)=1$ for some $i \in[1,2]$. In this case, say $u v$ is type $i$. Since there are three pairs of vertices in $T$ and only two types, two pairs of vertices in $T$ have the same type. Say $T=u v w$. Without loss of generality, $u v$ and $v w$ are both type 1 . That is, $\operatorname{dist}\left(u_{1}, v_{1}\right)=1$ and $\operatorname{dist}\left(v_{1}, w_{1}\right)=1$. Thus $\operatorname{dist}\left(u_{1}, w_{1}\right) \in\{0,2\}$, in which case $u w \notin E(G)$. This contradiction shows that $G$ contains no triangle.

Proposition 24. For all $\Delta \geqslant 42$ there is a triangle-free graph with diameter 3 , maximum degree at most $\Delta$, and at least $\left(2\left\lfloor\frac{\Delta+6}{12}\right\rfloor+4\right)^{3}$ vertices.

Proof. Let $p:=2\left\lfloor\frac{\Delta+6}{12}\right\rfloor+4$. Thus $p \geqslant 12$ is even. Let $H$ be the graph with vertex set $\mathbb{Z}_{p}$, where $a b \in E(H)$ whenever $\operatorname{dist}(a, b) \geqslant 3$. Observe that every pair of vertices in $H$ have a common neighbour (since $p \geqslant 12$ ).

Define a graph $G$ with vertex set $V(G):=\mathbb{Z}_{p}^{3}$. Thus $|V(G)|=p^{3}$. Let $\left(v_{1}, v_{2}, v_{3}\right)$ denote a vertex $v$ in $G$. For distinct vertices $v$ and $w$, define the vw-vector to be $(a, b, c)$, 
where $a \leqslant b \leqslant c$ and $\{a, b, c\}=\left\{\operatorname{dist}\left(v_{1}, w_{1}\right)\right.$, $\operatorname{dist}\left(v_{2}, w_{2}\right)$, $\left.\operatorname{dist}\left(v_{3}, w_{3}\right)\right\}$. Then $v w \in E(G)$ if and only if $a=0$ and $b=1$ and $c \geqslant 3$. Observe that $G$ is $6(p-5)$-regular, and $6(p-5) \leqslant \Delta$.

We now show that the distance between distinct vertices $v, w$ in $G$ is at most 3 . Consider the following cases for the $v w$-vector, where without loss of generality, $(a, b, c)=$ $\left(\operatorname{dist}\left(v_{1}, w_{1}\right), \operatorname{dist}\left(v_{2}, w_{2}\right), \operatorname{dist}\left(v_{3}, w_{3}\right)\right)$ :

Case $(0,0, \geqslant 1)$ : Let $u$ be a common neighbour of $v_{3}$ and $w_{3}$ in $H$. Then $\left(v_{1}+1, v_{2}, u\right)=$ $\left(w_{1}+1, w_{2}, u\right)$ is a common neighbour of $v$ and $w$.

Case $(0,1,1)$ : Then $\left(v_{1}+3, w_{2}, v_{3}\right)=\left(w_{1}+3, w_{2}, v_{3}\right)$ is a common neighbour of $v$ and $w$.

Case $(0,1,2)$ : Let $y$ be a common neighbour of $v_{2}$ and $w_{2}$ in $H$. Since $\operatorname{dist}\left(v_{3}, w_{3}\right)=2$, there is an element $z$ such that $\operatorname{dist}\left(v_{3}, z\right)=\operatorname{dist}\left(w_{3}, z\right)=1$. Then $\left(v_{1}, y, z\right)=\left(w_{1}, y, z\right)$ is a common neighbour of $v$ and $w$.

Case $(0,1, \geqslant 3)$ : Then $v$ and $w$ are adjacent.

Case $(0, \geqslant 2, \geqslant 2)$ : Since $\operatorname{dist}\left(v_{2}, w_{2}\right) \geqslant 2$, there is an element $y \in\left\{w_{2}-1, w_{2}+1\right\}$ such that $\operatorname{dist}\left(v_{2}, y\right) \geqslant 3$. Similarly, $\operatorname{dist}\left(w_{3}, z\right) \geqslant 3$ for some $z \in\left\{v_{3}-1, v_{3}+1\right\}$. Then $\left(v_{1}, y, z\right)$ is a common neighbour of $v$ and $w$.

Case $(1, \geqslant 1, \geqslant 1)$ : Since $v_{2} \neq w_{2}$, there is an element $u \in\left\{w_{2}, w_{2}+2, w_{2}-2\right\}$ such that $\operatorname{dist}\left(v_{2}, u\right) \geqslant 3$. Let $u^{\prime}$ be $\operatorname{such}$ that $\operatorname{dist}\left(u, u^{\prime}\right)=\operatorname{dist}\left(w_{2}, u^{\prime}\right)=1$. Let $z$ be a common neighbour of $v_{3}$ and $z_{3}$ in $H$. Then

$$
\left(v_{1}, v_{2}, v_{3}\right)\left(w_{1}, u, v_{3}\right)\left(w_{1}, u^{\prime}, z\right)\left(w_{1}, w_{2}, w_{3}\right)
$$

is a $v w$-path of length 3 .

Case $(\geqslant 2, \geqslant 2, \geqslant 2)$ : Since $p \geqslant 6$ and $\operatorname{dist}\left(v_{1}, w_{1}\right) \geqslant 2$, there exists $x \in \mathbb{Z}_{p}$ such that $\operatorname{dist}\left(w_{1}, x\right)=1$ and $\left.\operatorname{dist}\left(v_{1}, x\right) \geqslant 3\right)$. Similarly, there exists $y, z \in \mathbb{Z}_{p}$ such that $\operatorname{dist}\left(w_{2}, y\right)=1$ and $\operatorname{dist}\left(v_{2}, y\right) \geqslant 3$, and $\operatorname{dist}\left(v_{3}, z\right)=1$ and $\operatorname{dist}\left(w_{3}, z\right) \geqslant 3$. Then

$$
\left(v_{1}, v_{2}, v_{3}\right)\left(x, v_{2}, z\right)\left(w_{1}, y, z\right)\left(w_{1}, w_{2}, w_{3}\right)
$$

is a $v w$-path of length 3 .

Thus $G$ has diameter at most 3 .

Suppose on the contrary that $G$ contains a triangle $T$. For each edge $u v$ of $T$, we have $u_{i}=v_{i}$ for exactly one value of $i \in[1,3]$. In this case, say $u v$ is type $i$. First suppose that at least two of the edges in $T$ are the same type. Then all three edges in $T$ are the same type. Without loss of generality, $u_{1}=v_{1}=w_{1}$. Then the subgraph of $G$ induced by $\{u, v, w\}$ (ignoring the first coordinate) is a subgraph of the graph in Proposition 23, which is triangle-free. Now assume that all three edges in $T$ have distinct types. Without loss of generality, $u_{1}=v_{1}$ and $u_{2}=w_{2}$ and $v_{3}=w_{3}$. Since $u v \in E(G)$, without loss of generality, $\operatorname{dist}\left(u_{2}, v_{2}\right)=1$ and $\operatorname{dist}\left(u_{3}, v_{3}\right) \geqslant 3$. Thus $\operatorname{dist}\left(v_{2}, w_{2}\right)=1$ and $\operatorname{dist}\left(u_{3}, w_{3}\right) \geqslant 3$. Since $v w \in E(G)$ and $u_{1}=v_{1}$, we have $\operatorname{dist}\left(u_{1}, w_{1}\right)=\operatorname{dist}\left(v_{1}, w_{1}\right) \geqslant 3$. We have shown that $\operatorname{dist}\left(u_{1}, w_{1}\right) \geqslant 3$ and $u_{2}=w_{2}$ and $\operatorname{dist}\left(u_{3}, w_{3}\right) \geqslant 3$. Thus the $u w$-vector is $(0,3,3)$, implying $u w \notin E(G)$. This contradiction shows that $G$ is triangle-free. 
Finally, note that the graphs in Propositions 23 and 24 have bounded chromatic number. In Proposition 23, colour each vertex $v$ by $\left(v_{1} \bmod 2, v_{2} \bmod 2\right)$. For each edge $v w$, we have $\operatorname{dist}\left(v_{i}, w_{i}\right)=1$ for some $i$. Since $p$ is even, $v_{i} \not \equiv w_{i}(\bmod 2)$. Thus, this is a valid 4-colouring. In Proposition 24, colouring each vertex $v$ by $\left(v_{1} \bmod 2, v_{2} \bmod 2, v_{3} \bmod 2\right)$ gives an 8-colouring.

\section{Acknowledgement}

The case $k=2$ of Theorem 12 was proved in collaboration with Bruce Reed. Thanks Bruce.

\section{References}

[1] Norman Biggs. Algebraic graph theory. Cambridge Tracts in Mathematics, No. 67. Cambridge University Press, London, 1974. MR:0347649

[2] Hans L. Bodlaender. A partial $k$-arboretum of graphs with bounded treewidth. Theoret. Comput. Sci., 209(1-2):1-45, 1998. doi:10.1016/S0304-3975(97)00228-4

[3] Eduardo A. Canale And José Gómez. Asymptotically large $(\Delta, D)$-graphs. Discrete Appl. Math., 152(1-3):89-108, 2005. doi:10.1016/j.dam.2005.03.008 MR : 2174196

[4] Nicolats G. De Bruijn. A combinatorial problem. Nederl. Akad. Wetensch., Proc., 49:758-764, 1946. MR:0018142

[5] Charles Delorme. Large bipartite graphs with given degree and diameter. J. Graph Theory, 9(3):325-334, 1985. doi:10.1002/jgt.3190090304 MR:812399

[6] Erik D. Demaine, Fedor V. Fomin, Mohammad Taghi Hajiaghayi, and Dimitrios M. Thilikos. Bidimensional parameters and local treewidth. SIAM J. Discrete Math., 18(3):501-511, 2004/05. doi:10.1137/S0895480103433410 MR : 2134412

[7] Erik D. Demaine and Mohammad Taghi Hajiaghayi. Diameter and treewidth in minor-closed graph families, revisited. Algorithmica, 40(3):211-215, 2004. doi:10.1007/s00453-004-1106-1 MR:2080518

[8] Erik D. Demaine and Mohammad Taghi Hajiaghayi. Equivalence of local treewidth and linear local treewidth and its algorithmic applications. In Proc. 15th Annual ACM-SIAM Symposium on Discrete Algorithms (SODA '04), pp. 840-849. SIAM, 2004. http://dl.acm.org/citation. cfm?id=982792.982919

[9] Reinhard Diestel. Graph theory, vol. 173 of Graduate Texts in Mathematics. Springer-Verlag, Berlin, 3rd edn., 2005. http://diestel-graph-theory.com/ 
[10] Vida Dujmović, Pat Morin, and David R. Wood. Layered separators in minor-closed families with applications, 2014. arXiv:1306.1595

[11] David Eppstein. Diameter and treewidth in minor-closed graph families. Algorithmica, 27(3-4):275-291, 2000. doi:10.1007/s004530010020 MR:1759751

[12] Michael Fellows, Pavol Hell, and Karen Seyffarth. Large planar graphs with given diameter and maximum degree. Discrete Appl. Math., 61(2):133-153, 1995. doi:10.1016/0166-218X (94)00011-2

[13] Michael Fellows, Pavol Hell, and Karen Seyffarth. Constructions of large planar networks with given degree and diameter. Networks, 32(4):275-281, 1998.

[14] Miguel A. Fiol, José L. A. Yebra, and Ignacio Alegre. Line digraph iterations and the $(d, k)$ digraph problem. IEEE Trans. Comput. C-33:400-403, 1984. doi:10.1109/TC.1984.1676455

[15] Cyril Gavoille, David Peleg, André Raspaud, and Eric Sopena. Small $k$-dominating sets in planar graphs with applications. In Graph-theoretic concepts in computer science (WG '01), vol. 2204 of Lecture Notes in Comput. Sci., pp. 201-216. Springer, 2001. doi:10.1007/3-540-45477-2_19 MR:1905633

[16] Irving J. Good. Normal recurring decimals. J. London Math. Soc., 21:167-169, 1946. doi:10.1112/jlms/s1-21.3.167

[17] Martin Grohe. Local tree-width, excluded minors, and approximation algorithms. Combinatorica, 23(4):613-632, 2003. doi:10.1007/s00493-003-0037-9 MR : 2046826

[18] Charles C. Harner And Roger C. Entringer. Arc colorings of digraphs. J. Combinatorial Theory Ser. B, 13:219-225, 1972. doi:10.1016/0095-8956(72) 90057-3 MR:0313101

[19] Pavol Hell and Karen Seyffarth. Largest planar graphs of diameter two and fixed maximum degree. Discrete Math., 111(1-3):313-322, 1993. doi : 10.1016/0012-365X (93) 90166-Q

[20] Hiroyuki Kawai and Yukio Shibata. The chromatic number and the chromatic index of de Bruijn and Kautz digraphs. IEICE Trans Fundam Electron Commun Comput Sci, E85-A(6):1352-1358, 2002. http://sciencelinks.jp/j-east/ article/200215/000020021502A0487232.php

[21] Alexandr V. Kostochka. The minimum Hadwiger number for graphs with a given mean degree of vertices. Metody Diskret. Analiz., 38:37-58, 1982. MR:0713722 $\mathrm{Zbl}: 0544.05037$ 
[22] Mirka Miller AND Jozef Širáñ. Moore graphs and beyond: A survey of the degree/diameter problem. Electron. J. Combin., DS14, 2013. http://www. combinatorics.org/ojs/index.php/eljc/article/view/DS14

[23] Bojan Mohar and Carsten Thomassen. Graphs on surfaces. Johns Hopkins University Press, Baltimore, U.S.A., 2001.

[24] Crispin St. J. A. Nash-Williams. Decomposition of finite graphs into forests. J. London Math. Soc., 39:12, 1964. doi:10.1112/jlms/s1-39.1.12

[25] Eran Nevo, Guillermo Pineda-Villabicencio, and David R. Wood. On the maximum order of graphs embedded in surfaces, 2013. arXiv:1312.1753

[26] Bruce A. ReED. Tree width and tangles: a new connectivity measure and some applications. In Surveys in combinatorics, vol. 241 of London Math. Soc. Lecture Note Ser., pp. 87-162. Cambridge Univ. Press, 1997. doi:10.1017/CB09780511662119.006

[27] Jana Šiagiová and Rinovia Simanjuntak. A note on a Moore bound for graphs embedded in surfaces. Acta Math. Univ. Comenian. (N.S.), 73(1):115-117, 2004. MR : 2076049

[28] Andrew Thomason. An extremal function for contractions of graphs. Math. Proc. Cambridge Philos. Soc., 95(2):261-265, 1984. doi:10.1017/S0305004100061521 MR:0735367 Zbl:0551.05047

[29] Andrew Thomason. The extremal function for complete minors. J. Combin. Theory Ser. B, 81(2):318-338, 2001. doi:10.1006/jctb.2000.2013 MR:1814910 $\mathrm{Zbl}: 1024.05083$

[30] Serge A. Tishchenko. Maximum size of a planar graph with given degree and even diameter. European J. Combin., 33(3):380-396, 2012. doi:10.1016/j.ejc.2011.09.005

[31] Serge A. Tishchenko. $N$-separators in planar graphs. European J. Combin., 33(3):397-407, 2012. doi:10.1016/j.ejc.2011.09.003

[32] Fu Ji Zhang and Guo Ning Lin. On the de Bruijn-Good graphs. Acta Math. Sinica, 30(2):195-205, 1987. MR:891925 